\title{
Facile Growth of Multi-twined Au Nanostructures
}

\author{
RAJ KUMAR BERA ${ }^{\mathrm{a}}$, ASIM BHAUMIK ${ }^{\mathrm{b}}$ and C RETNA RAJ ${ }^{\mathrm{a}, *}$ \\ aDepartment of Chemistry, Indian Institute of Technology, Kharagpur 721 308, India \\ ${ }^{b}$ Department of Materials Science and Centre for Advanced Materials, Indian Association for the Cultivation \\ of Science, Jadavpur, Kolkata 700 032, India \\ e-mail: crraj@chem.iitkgp.ernet.in
}

MS received 9 July 2015; revised 21 September 2015; accepted 5 October 2015

\begin{abstract}
We describe a facile growth of chain-like Au nanostructures and their spontaneous transformation to multi-twined nanostructure using a mild reducing agent bisphenol A (BPA). The growth Au nanostructures involves the chemical reduction of $\mathrm{HAuCl}_{4}$ by BPA in the presence of cetyltrimethylammonium bromide $(\mathrm{CTAB})$ as capping agent in alkaline condition without any seeds. Wire and chain-like Au nano-network structures with diameter in the range of 4 to $9 \mathrm{~nm}$ are obtained in the initial stage of the reaction. These chainlike nanostructures undergo spontaneous transformation into multi-twined nanostructures within $24 \mathrm{~h}$. These nanocrystalline multi-twined structures have an average size of 80-90 nm. X-ray and selected area electron diffraction measurements reveal that the Au nanoparticles have (111), (200), (220) and (311) planes of a face centered cubic structure. High resolution transmission electron microscopic measurement shows that the nanostructures are mainly composed of (111) lattice plane with twin boundaries. The concentration of $\mathrm{HAuCl}_{4}, \mathrm{BPA}$ and CTAB has pronounced effect in the growth of nanostructures. The multi-twined nanostructures are highly stable at room temperature over a period of one month and can be used for catalytic applications.
\end{abstract}

Keywords. Bisphenol A; chain-like Au nano-network; spontaneous transformation; multi-twined Au nanostructures.

\section{Introduction}

Shape controlled synthesis of metal and semiconducting nanostructures is one of the major areas of research in nanoscience and nanotechnology, as the shape and surface structure of these materials have great control over their optical, electronic, and catalytic properties. The tailor-made nanomaterials have potential application in various areas such as, catalysis, ${ }^{1}$ biosensing, ${ }^{2}$ photovoltaics, ${ }^{3}$ nanoelectronics, etc. ${ }^{4}$ The growth of transition metal nanostructures is of special interest for analytical chemists owing to their unique optical and electronic properties. For instance, the fascinating optical properties of Au nanoparticles attracted significant interest in the development of sensing/biosensing methodologies. The anisotropic Au nanostructures are known to display transverse and longitudinal surface plasmon resonance and have been used as a potential substrate for surface enhanced Raman scattering, ${ }^{5}$ excellent catalyst for various industrially important reactions, ${ }^{1}$ and a color indicating reagent for the sensing of biomolecules. ${ }^{2}$ The properties of these nanoparticles can be tuned according to the requirement by tailoring

\footnotetext{
*For correspondence
}

their shape and surface structure. Various attempts have been made to tailor the shape of the metal particles by changing the synthetic methodology. Au nanoparticle of different shapes such as nanocube, rod, wire, prism, plate, belt, star, etc. ${ }^{6-23}$ were traditionally synthesized using suitable reducing agents, surfactants, polymers, seeds, and templates. Murphy's group has extensively studied the seed-mediated growth of anisotropic $\mathrm{Au}$ nanostructures using cetyltrimethylammonium bromide $(\mathrm{CTAB})$ as a capping agent in aqueous solution. ${ }^{24,25}$ Xia's group pioneered the synthesis of anisotropic metal nanostructures by polyol route. ${ }^{26,27}$ Sastry and co-workers exploited biological extracts for the synthesis of anisotropic $\mathrm{Au}$ and $\mathrm{Ag}$ nanostructures. ${ }^{28-31}$ The capping and reducing agents play a vital role in controlling the shape and morphology of the metal particles. Umar and Oyama have reported the high yield synthesis of branched $\mathrm{Au}$ nanostructures using binary mixture of CTAB and hexamethylenetetramine by the seed-mediated growth approach. ${ }^{32}$ Liz-Marzan reported the synthesis of thorned nanowires by the seed-mediated approach in non-aqueous solvent. ${ }^{33}$ Tsai and co-workers recently reported the controlled synthesis of Au nanopyramids, nanostars and nanorods by seed-mediated growth. ${ }^{34}$ In the popular seed-mediated growth approach, ascorbic acid is traditionally used as 
a mild reducing agent and the growth of Au nanostructures was induced by $\mathrm{NaOH}$ or $\mathrm{Au}$ nanoseeds and the capping agent CTAB is known to regulate the growth of nanostructures. The nature of reducing agent plays important role in controlling the growth of nanoparticles. The morphology, shape and size can be regulated by selecting appropriate reducing agents. Small organic molecules having suitable functional groups and reduction potential can serve as ideal reducing agents. ${ }^{35-39}$ The mild reducing agents are of special importance as the controlled reduction of metal precursors can yield nanoparticles of desired shape and morphology. In an effort to develop a new synthetic method, herein we demonstrate the use of a mild reducing agent bisphenol A (BPA) and the growth of chain-like Au nanostructures and their spontaneous self-transformation into thermodynamically stable multi-twined nanostructures.

\section{Experimental}

\subsection{Materials}

$\mathrm{HAuCl}_{4}$ and BPA were purchased from Sigma-Aldrich. Analytical grade $\mathrm{CTAB}$ and $\mathrm{NaOH}$ were purchased from Merck. All other reagents used in this investigation were of analytical grade ( $>99 \%$ purity) and used without further purification.

\subsection{Instrumentation}

Electronic absorption spectral measurements were performed using CARY 5000 UV-Visible-NIR spectrophotometer. Transmission electron microscopy (TEM) images were acquired with JEOL JEM-2010 microscopes at an operating voltage of $200 \mathrm{kV}$. X-ray diffraction (XRD) patterns of the samples were collected using a Philips X'pert PRO R-ray diffraction unit using $\mathrm{Ni}$ filtered $\mathrm{Cu} \mathrm{K} \alpha(\lambda=1.54 \AA$ A $)$ radiation. Dynamic Light Scattering (DLS) measurement was performed with Malvern Nano ZS instrument employing a $4 \mathrm{~mW}$ He-Ne laser $(\lambda=632.8 \mathrm{~nm})$. Electrochemical experiments were performed with CHI643B electrochemical analyzer (CHI, Austin, Texas). Two-compartment, three electrode electrochemical cell with glassy carbon (GC) working, $\mathrm{Ag} / \mathrm{AgCl}(3 \mathrm{M} \mathrm{KCl})$ reference and $\mathrm{Pt}$ wire auxiliary electrodes were used in the electrochemical studies.

\subsection{Synthesis of colloidal nanoparticles}

In a typical synthesis, $40 \mu \mathrm{L}$ of $\mathrm{HAuCl}_{4}(30 \mathrm{mM})$ was mixed with $1 \mathrm{~mL}$ of aqueous CTAB $(20 \mathrm{mM})$ and 1.5
$\mathrm{mL}$ of Millipore water under constant stirring to obtain bright orange colored solution. To this solution, 400 $\mu \mathrm{L}$ of BPA $(0.3 \mathrm{mM})$ was added. After the addition of BPA the orange color of the mixture changed initially to pale yellow and then it became colorless within 30 min. Then $15 \mu \mathrm{L}$ of $\mathrm{NaOH}(1 \mathrm{M})$ was added to the reaction vessel to induce the growth of $\mathrm{Au}$ nanoparticles. Greenish-blue color was observed immediately after the addition of $\mathrm{NaOH}$, indicating the formation of Au nanoparticles. The color of the reaction mixture changed from greenish-blue to blue within $1 \mathrm{~h}$ and it turned to purple-red after $24 \mathrm{~h}$. The final concentration of $\mathrm{HAuCl}_{4}, \mathrm{CTAB}, \mathrm{BPA}$, and $\mathrm{NaOH}$ in the reaction vessel was $0.4,6.6,0.04$ and $5 \mathrm{mM}$, respectively. The resulting colloidal nanoparticle sample was stored at room temperature.

\section{Results and Discussion}

\subsection{Synthesis and characterization of $A u$ nanosructures}

The synthesis of multi-twined $\mathrm{Au}$ nanostructures involves the reduction of $\mathrm{HAuCl}_{4}$ by BPA in the presence of $\mathrm{CTAB}$ and $\mathrm{NaOH}$ in aqueous solution. The growth of $\mathrm{Au}$ nanoparticles was monitored in real time by UV-Vis spectral measurements. Figure 1 displays the time-dependent spectral response obtained during the growth of Au nanoparticles. In the initial stage (after addition of $\mathrm{NaOH}$ ), a broad absorption band at $\sim 600$ $\mathrm{nm}$ was observed. The appearance of broad band may be ascribed to the interaction of smaller particles produced at initial stage of the reaction. Gradual increase in the absorbance at $600 \mathrm{~nm}$ associated with a slight blue shift was observed during the progress of the reaction, suggesting the further growth of nanoparticles.

Moreover, a significant raise in the absorbance in the near-IR region was also noticed during the first $1 \mathrm{~h}$ of the reaction (figure 1B). The band at $\sim 600 \mathrm{~nm}$ continues to grow with time and shifted to shorter wavelength side. However, the absorbance in the near-IR region starts to decrease after $1 \mathrm{~h}$ of the reaction and $\sim 41 \%$ decrease in the absorbance was observed after $12 \mathrm{~h}$. The change in the spectral profile continued and finally the absorption band stayed off at $540 \mathrm{~nm}$ after 24 h. It should be pointed out here that $\sim 88 \%$ decrease in the absorbance in the near-IR region (at $1350 \mathrm{~nm}$ ) was observed (figure 1B), suggesting the structural transformation of the nanostructures. No further change in the spectral profile was noticed after 1 day; the absorbance and band position of the nanoparticle remained same up to 1 month. The spectral profiles obtained at different stages of the reaction suggest that shape/size of the 


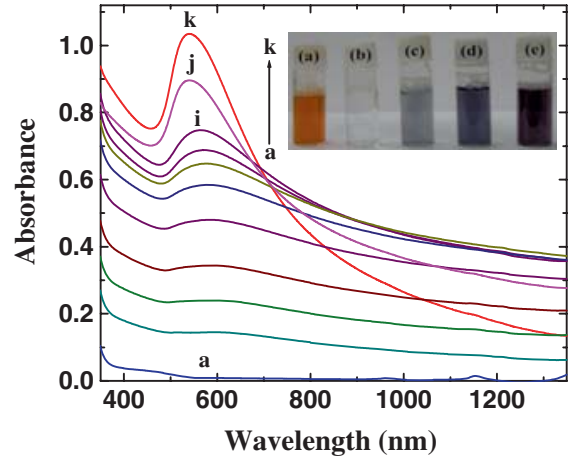

(a)

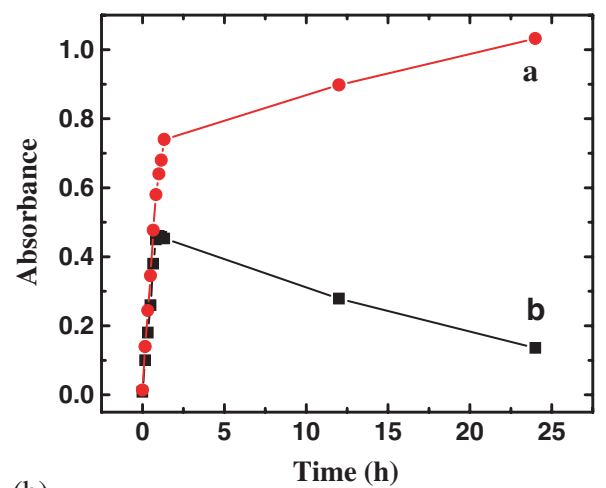

(b)

Figure 1. (A) Time-dependant absorption spectra illustrating the growth of $\mathrm{Au}$ nanoparticles. The spectra were recorded at regular time interval of $10 \mathrm{~min}$ (a-i), at 12 $\mathrm{h}(\mathrm{j})$ and after $24 \mathrm{~h}(\mathrm{k})$. Inset shows the phtograph of (a) $\mathrm{HAuCl}_{4}-\mathrm{CTAB}$ mixture and (b-e) nanoparticle at different reaction times: (b) 0; (c) 0.16; (d) 1 and (e) 24 h. (B) Plot of absorbance vs time at (a) $540 \mathrm{~nm}$ (b) $1350 \mathrm{~nm}$.

nanoparticle changes with time. The full width at half maximum (FWHM) of the spectrum obtained at $1 \mathrm{~h}$ is significantly larger than those obtained at $24 \mathrm{~h}$. The SPR band width for the particles in the extrinsic size region (>25 nm size) increases with increasing size. ${ }^{40}$ The extinction spectrum of larger nanoparticles is dominated by higher oscillation mode and the bandwidth is known to increase with size and the broadening of SPR is associated to the retardation effect. ${ }^{40}$ In the present case, the decrease in the FWHM of SPR band for the nanoparticles obtained at $24 \mathrm{~h}$ suggests the transformation of larger nanoparticles during the course of the reaction.

TEM measurements have been performed to know the size and morphology of the nanoparticles at different stages of the reaction. Figure 2 is the TEM images of the nanoparticles obtained at $1 \mathrm{~h}$ of the reaction. TEM images obtained at $1 \mathrm{~h}$ of the reaction show chain and wire-like nanostructures. The chain-like nanostructures do not have uniform diameter and varies from 4 to 9 $\mathrm{nm}$. The length of the chain is not measurable. The magnified image of the nanoparticle reveals that the nanostructures have 2-dimensional network (figure 2). These nanostructures are interconnected by small spherical or ellipsoidal nanoparticles. The selected area electron diffraction (SAED) pattern of the nanostructure can be indexed to (111), (200), (220) and (311) planes of face centered cubic lattice (fcc) (figure 2). HRTEM image reveals the fringe spacing of 2.39 $\AA$, corresponding to the interplaner distance of (111) planes (figure 2). On the other hand, the nanoparticles obtained at $24 \mathrm{~h}$ are multi-twined and have an average

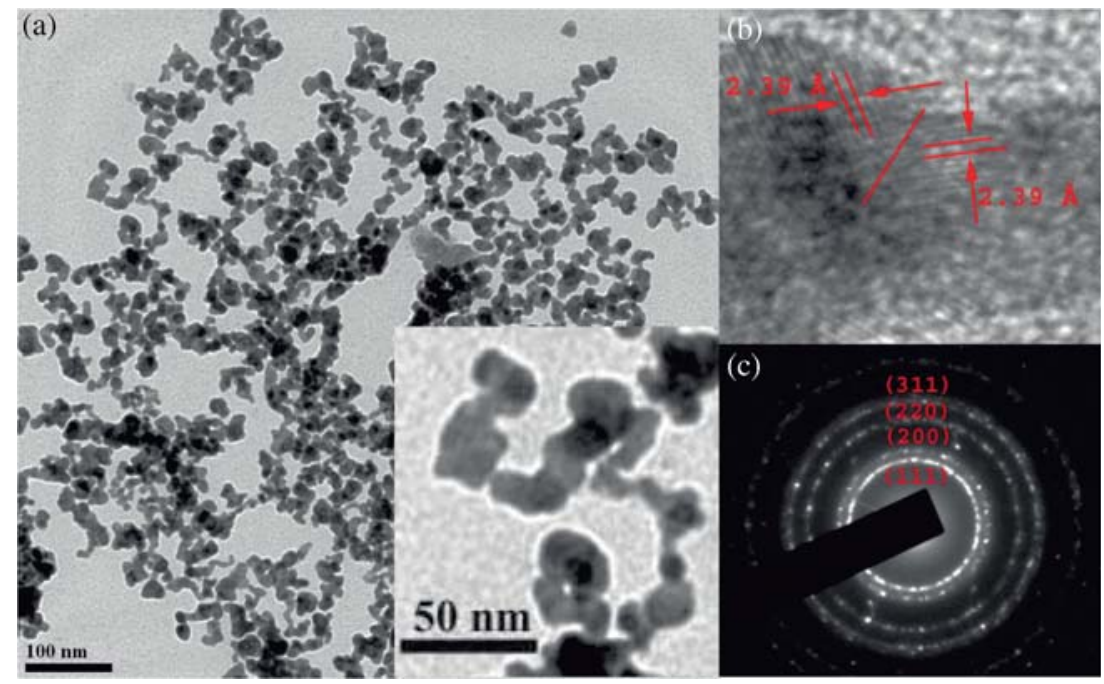

Figure 2. TEM images of the chain-like nanoparticle obtained at $1 \mathrm{~h}$ of the reaction. Inset is the magnified TEM image. (b) HRTEM image showing the fringe spacing and (c) SAED pattern. 

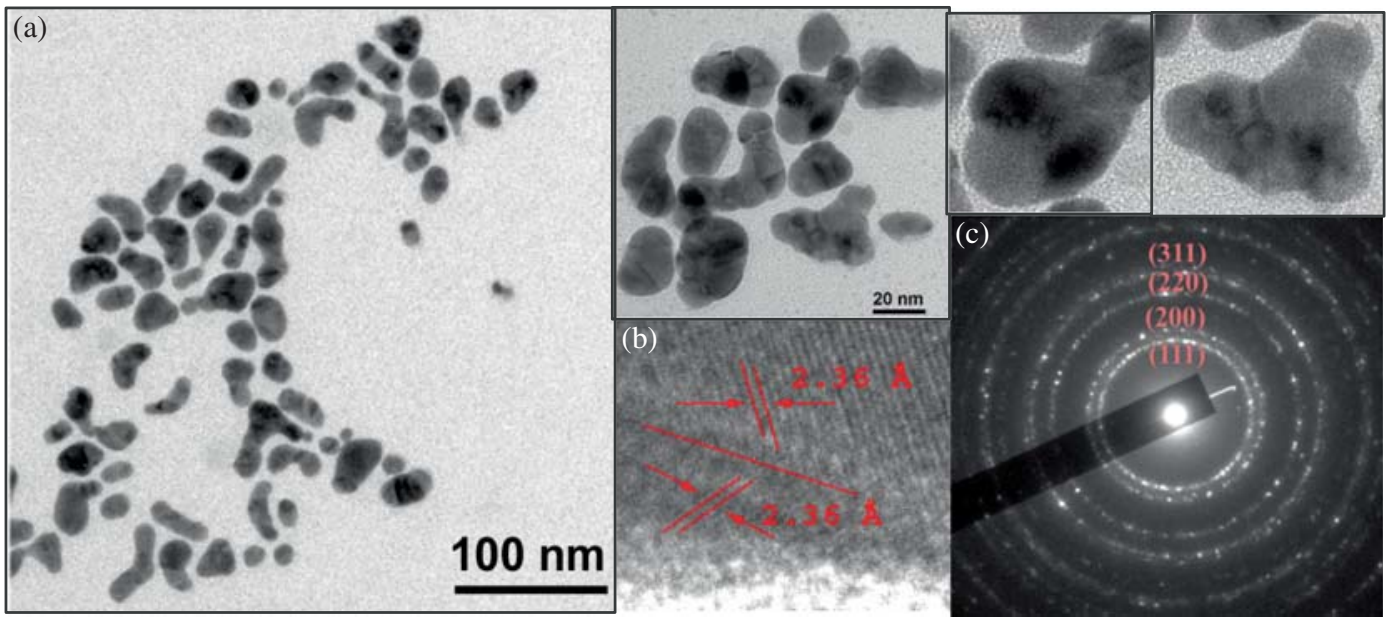

Figure 3. TEM images of the nanoparticles obtained at $24 \mathrm{~h}$ of the reaction. Inset shows the magnified image of the nanoparticles. The tadpole-like structures are shown separately. (b) HRTEM image and (c) SAED pattern obtained for the tadpole-like structure.

size of $90 \mathrm{~nm}$ (figure 3 ). The chain and wire-like nanostructures formed at $1 \mathrm{~h}$ of the reaction spontaneously transformed into nanoparticles of other shapes within $24 \mathrm{~h}$. It has been demonstrated earlier that the thermodynamically unstable branched $\mathrm{Au}$ nanoparticles transformed into spherical nanoparticles. ${ }^{41}$ The $\mathrm{Au}$ atoms of the branched nanocrystal rearrange themselves to form thermodynamically more stable spherical nanoparticles. ${ }^{41}$ Teng and Yang have observed the transition of branched Pt nanocrystal to spherical nanostructures. ${ }^{42}$ In the present case, the chain and wire-like nanostructures undergo spontaneous selftransformation and yield thermodynamically stable multi-twined nanostructures. The surface structure of branched $\mathrm{Au}$ nanostructures was further characterized by HRTEM measurement. As shown in figure 2 the nanocrystals have twin boundaries. The lattice fringe spacing was determined to be $2.36 \AA$ corresponding to the interplaner spacing between (111) planes (figure 3). The SAED pattern can be indexed to (111), (200), (220) and (311) plane of fcc structure of Au (figure 3). The existence of such planes was further confirmed by XRD measurement (Figure S1 in Supporting information). To understand the transformation of chain-like structure to multi-twined structure, TEM measurement was done after $12 \mathrm{~h}$ of the reaction. It was observed that the chain-like network structures spontaneously break into small chain-like structure (Figure S2 in SI). Note that the length and size of the nano-chains is relatively smaller than that obtained at the initial stage of the reaction. After $24 \mathrm{~h}$, this small chain-like structures fur ther transform into multi-twined structures (figure 3 ). It is known that the peak position and intensity of these spectral bands depends on the particle shape and size/aspect ratio. In our case, the nanoparticles show only one band. As described earlier, the nanoparticle shows significantly high absorbance at near-IR region (figure 1), though no distinct band was obtained. Unlike the anisotropic nanostructures, our multi-twined nanostructures do not exhibit both transverse and longitudinal bands, probably due to the polydispersity of the nanostructures. ${ }^{20}$ We suggest that the superposition of the longitudinal bands corresponding to the nanoparticles of various size/aspect ratios could be a reason for the absence of a separate band. The dynamic light scattering measurement for the nanoparticles obtained after $24 \mathrm{~h}$ indicates that the particles have the size distribution between 40 and $150 \mathrm{~nm}$ (figure S3 in SI). The majority of particles have the size of $90 \mathrm{~nm}$, which is in good agreement with the TEM measurement. It is worth mentioning here that although several small organic molecules have been used as mild reducing agents for the synthesis of Au nanoparticles of various morphologies (table S1 in SI), the growth of chain-like nanostructures and the spontaneous self-transformation to multi-twined nanostructures have not been observed.

\subsection{Effect of the concentration of CTAB, $\mathrm{HAuCl}_{4}$ and $B P A$}

The growth of the nanoparticle is highly sensitive to the concentration of capping agent $\mathrm{CTAB}, \mathrm{HAuCl}_{4}$ and reducing agent BPA. The concentration of CTAB strongly influences the formation of nanoparticles. It is generally expected that the high concentration of CTAB slows down the reduction of metal precursor and controls the kinetics of the growth of nanostructures. In our case, the reaction is very fast at lower 
concentration of CTAB and we could observe the rapid formation of nanoparticle by naked eye. For instance, the formation of nanoparticle was observed within 30 min at the CTAB concentration of $0.12 \mathrm{mM}$. The colloidal nanoparticle obtained at low CTAB concentration shows single intense surface plasmon band $\sim 530$ $\mathrm{nm}$. The band position and absorbance do not change with time. Figure 4A displays the UV-Vis spectra of the nanoparticles obtained at different concentrations of CTAB. All spectral measurements were performed after $1 \mathrm{~h}$ of the reaction. Following spectral features were obtained while decreasing the concentration of CTAB from 6.6 to $0.7 \mathrm{mM}$ : (i) significant increase in the absorbance of the band at $575 \mathrm{~nm}$ and decrease in the absorbance in the near-IR region, (ii) blue shift (10$15 \mathrm{~nm}$ ) in the surface plasmon band position, and (iii) decrease in the FWHM of the plasmon band. After $24 \mathrm{~h}$ of the reaction all the samples show single band $\sim 539 \mathrm{~nm}$ with very low absorbance in the near-IR region (figure 4B). However, the spectral feature of the nanoparticle obtained at high concentration (6.6 $\mathrm{mM}$ ) of CTAB is distinct from others (figure 4B). The absorbance at the near-IR region and the FWHM are relatively high with respect to the others, indicating that the concentration of CTAB has major role in controlling the shape of the nanoparticle. High concentration of CTAB favor the growth of tadpole-like structure as the rate of reduction is significantly low. It is believed that CTAB slows down the reduction process and controls the shape of nanoparticles. The spectral pattern obtained at low concentration of CTAB (figure S4 in SI) suggests the formation of spherical nanostructures. The formation of spherical nanoparticle at low concentration of CTAB was confirmed by TEM measurements (figure S5 in SI).

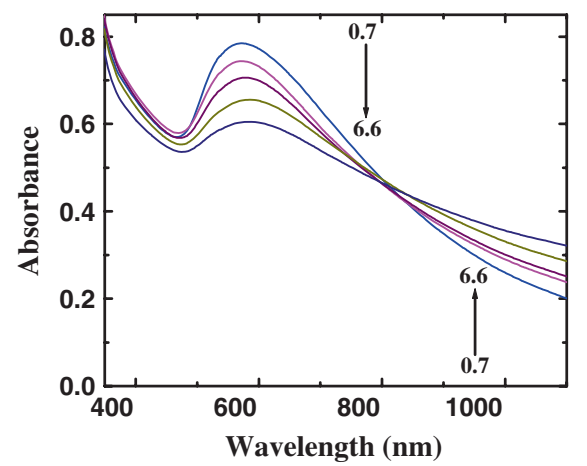

(a)

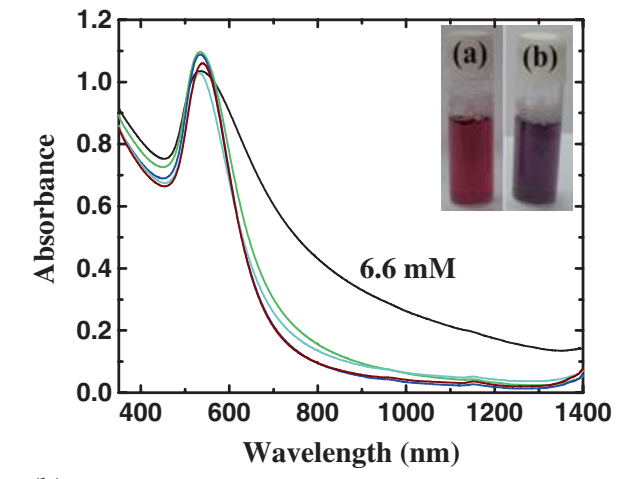

(b)

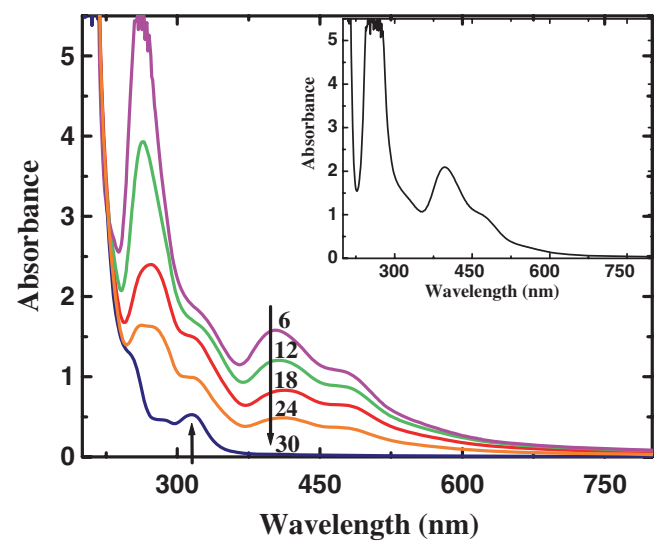

Figure 5. The absorption spectra obtained for $\mathrm{Au}(\mathrm{III})$ CTAB complex in the presence of BPA $\left(\mathrm{HAuCl}_{4}: 0.4 \mathrm{mM}\right.$, CTAB: $6.6 \mathrm{mM}$, and BPA: $40 \mu \mathrm{M})$ at $6 \mathrm{~min}$ time interval. Inset shows the absorption spectra for the $\mathrm{Au}(\mathrm{III})-\mathrm{CTAB}$ complex in the absence of BPA.

tion of $\mathrm{HAuCl}_{4}$ show a band at $530 \mathrm{~nm}$ with FWHM of $\sim 67 \mathrm{~nm}$. The band position shifts to longer wavelength side and the FWHM increases while increasing the concentration of $\mathrm{HAuCl}_{4}$ (Figure S6). The increase of FWHM suggests that the shape/morphology changes with the concentration $\mathrm{HAuCl}_{4}$. Further increase in the concentration does not bring any change in the spectral pattern. Blue shift of the surface plasmon band position and increase in the absorbance were observed when the concentration of BPA was increased from 10 to $240 \mu \mathrm{M}$. The spectrum obtained at high concentration of BPA is narrow and is similar to those of spherical nanoparticle (Figure S7). The effect of very high concentration of BPA was not tested due to its limited solubility in aqueous solution. The decrease in FWHM at

Figure 4. UV-Vis spectra of the nanoparticles obtained at different concentrations $(0.7,0.9,1.6,3.6$ and $6.6 \mathrm{mM})$ of CTAB at (A) $1 \mathrm{~h}$ and (B) $24 \mathrm{~h}$ of the reaction. Inset in Figure $4 \mathrm{~B}$ shows the photograph of the nanoparticles obtained at CTAB concentration of (a) 0.7 (b) $6.6 \mathrm{mM}$. 


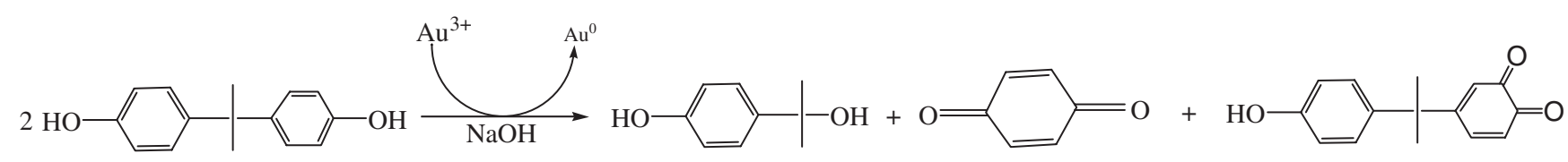

Scheme 1. Proposed reaction mechanism for the reduction of $\mathrm{Au}(\mathrm{III})$ to $\mathrm{Au}(0)$ by $\mathrm{BPA}$.

high concentration evidences the formation of smaller particles.

\subsection{Mechanistic pathway for the reduction of $A u(I I I)$ by $B P A$}

$\mathrm{HAuCl}_{4}$ in aqueous solution displays absorption band $\sim 290 \mathrm{~nm}$ due to metal-to-ligand charge transfer. ${ }^{43}$ On the other hand, CTAB-HAuCl${ }_{4}$ mixture shows three bands at 260, 407 and $475 \mathrm{~nm}$ (figure 5, inset) and are ascribed to the complexation of anionic $\mathrm{AuCl}_{4}^{-}$with cationic surfactant. ${ }^{44}$ The absorbance of these bands gradually decreases upon the addition of BPA and the band disappears completely in $30 \mathrm{~min}$. The bright orange color of the solution became colorless, indicating the reduction of $\mathrm{Au}(\mathrm{III})$ to $\mathrm{Au}(\mathrm{I})$ by BPA. A new absorption band at $318 \mathrm{~nm}$, presumably due to the oxidation product of BPA, was observed after 30 min (vide infra). The color of the solution turned to greenish-blue immediately after the addition of a small quantity of $\mathrm{NaOH}$, confirming the growth of nanoparticles. These observations are similar to the reduction of $\mathrm{HAuCl}_{4}$ CTAB complex by ascorbic acid. ${ }^{44,45}$ Electrochemical oxidation of BPA in neutral aqueous solution displays a broad voltammetric peak at $0.58 \mathrm{~V}(\mathrm{vs} \mathrm{Ag} / \mathrm{AgCl})$. The peak potential shifts to less positive side while increasing the solution $\mathrm{pH}$. As the oxidation peak potential of BPA falls within the oxidation and reduction potential of $\mathrm{Au}$ (figure S8 in SI), the reduction of $\mathrm{HAuCl}_{4}$ to metallic Au by BPA is thermodynamically feasible. It is worth pointing out that addition of alkaline BPA to the $\mathrm{CTAB}-\mathrm{HAuCl}{ }_{4}$ mixture immediately turns the bright orange color to colorless and then to greenish blue color. The immediate disappearance of the orange color of the CTAB-HAuCl${ }_{4}$ mixture upon the addition of alkaline BPA suggests that BPA has higher reducing strength in alkaline condition. It has been demonstrated recently that the reducing strength of hydroxyl reducing agents depends on the solution $\mathrm{pH}$ at which the reaction is conducted. ${ }^{46}$ The acid dissociation constant $\left(\mathrm{p} K_{\mathrm{a}}\right)$ of BPA is reported to be $9.46^{47}$ and it deprotonates in alkaline $\mathrm{pH}$. The $\mathrm{pH}$ of the solution alters the oxidation potential of BPA and can favor the formation of $\mathrm{Au}$ nanoparticles. The oxidation of BPA is known to yield different products such as 1,4-benzoquinone and monoquinone derivatives of BPA, etc., depending on the experimental conditions. ${ }^{48-50}$ The absorption spectrum of BPA in neutral aqueous solution shows two bands at 225 and $275 \mathrm{~nm}$; these bands appear at 245 and 297 $\mathrm{nm}$ in alkaline solution (figure S9). The oxidation product 1,4-benzoquinone is known to display one strong absorption band at $\sim 244 \mathrm{~nm}$ and a weak band at $\sim 330$ $n m .{ }^{48}$ On the other hand, the monoquinone derivative of BPA shows two bands at $\sim 394$ and $\sim 270 \mathrm{~nm}^{48-50}$ The absorption spectra recorded for the $\mathrm{Au}(\mathrm{III})-\mathrm{CTAB}$ complex in the presence of BPA in neutral solution at different time intervals points out that $\mathrm{Au}$ (III) is reduced to $\mathrm{Au}(\mathrm{I})$ (figure 5). The new absorption bands observed at 249 and $318 \mathrm{~nm}$ (figure 5) can be attributed to 1,4benzoquinone. To delve into the detailed mechanism for the reduction of $\mathrm{Au}(\mathrm{III})$ to $\mathrm{Au}(0)$, the colloidal nanoparticle was centrifuged at the speed of $14000 \mathrm{rpm}$ in an ultra centrifuge and the supernatant was subjected to spectral measurements. Three distinct spectral bands 251, 314 and $400 \mathrm{~nm}$ were observed for the supernatant (figure S10 in SI). These bands are ascribed to the oxidation products 1,4-benzoquinone and monoquinone derivative of BPA. Thus we suggest that reduction of $\mathrm{Au}(\mathrm{III})$ to $\mathrm{Au}(0)$ occurs according to the mechanism proposed in scheme 1 . It should be mentioned here that the oxidation of BPA can also give other byproducts which are not identified in our spectral measurements. The formation of other such byproducts cannot be ruled out and the identification of other product(s) is beyond the scope of the present investigation.

\section{Conclusions}

We have for the first time demonstrated that BPA can be used as a mild reducing agent for the synthesis of multi-twinned $\mathrm{Au}$ nanoparticle in aqueous solution. Self-transformation of chain and wire-like $\mathrm{Au}$ nanostructure into multi-twined structures was observed. The thermodynamically unstable wire-like nanostructures formed in the initial stage transform with time. The multi-twined nanostructures obtained after $24 \mathrm{~h}$ are stable for months. The concentration of CTAB, $\mathrm{HAuCl}_{4}$ and BPA controls the growth of $\mathrm{Au}$ nanostructure. The reduction of $\mathrm{Au}(\mathrm{III})$ to $\mathrm{Au}(0)$ proceeds through formation of $\mathrm{Au}(\mathrm{I})$ species. The possible oxidation products of BPA were identified by 
spectral measurements. The phenolic endocrine disruptors can be conveniently used for the synthesis of metal nanoparticles of different shapes and morphology.

\section{Supplementary Information}

XRD profile obtained for the $\mathrm{Au}$ nanoparticles of tadpole-like structure, TEM image at $12 \mathrm{~h}$ of the reaction, DLS plot, absorption spectra for the nanoparticles obtained at various concentrations of $\mathrm{HAuCl}_{4}$, and BPA, low concentration of CTAB, cyclic voltammogram of BPA, absorption spectra of supernatant solution, BPA in neutral and alkaline solution and spectra obtained for the optical sensing at lower concentration of BPA are shown in figures S1 - S10 and table S1. Supplementary Information is available at www.ias.ac. in/chemsci.

\section{Acknowledgements}

This work was financially supported by Department of Science and Technology and Indian Institute of Technology. R. K. Bera acknowledges the receipt of UGC fellowship.

\section{References}

1. Moshfegh A Z 2009 J. Phys. D: Appl. Phys. 42233001

2. Wang H, Yang R, Yang L and Tan W 2009 ACS Nano 3 2451

3. Kamat P V 2002 J. Phys. Chem. B 1067729

4. Tu C-C, Li Y-K, Chen T-M and Wu C-Y 2006 IEEE Transact. Nanotechnol. 5284

5. Nie S and Emory S R 1997 Science 2751102

6. Jin R, Egusa S and Scherer N F 2004 J. Am. Chem. Soc. 1269900

7. Jana N, Gearheart L and Murphy C 2001 J. Phys. Chem. B 1054065

8. Kim F, Song J H and Yang P 2002 J. Am. Chem. Soc. 12414316

9. Kou X, Zhang S, Tsung C-K, Yang Z, Yeung M H, Stucky G D, Sun L, Wang J and Yan C 2007 Chem. Eur. J. 132929

10. Imanishi A, Masaki T and Kuwabata S 2009 Chem. Commun. 1775

11. Iqbal M, Chung Y I and Tae G Y 2007 J. Mater. Chem. 17335

12. Kim J-U, Cha S-H, Shin K, Jho J Y and Lee J-C 2004 Adv. Mater. 16459

13. Guo Z, Fan X, Liu L, Bian Z, Gu C, Zhang Y, Gu N, Yang D and Zhang J 2010 J. Colloid Interface Sci. 348 29

14. Halder A and Ravishankar N 2007 Adv. Mater. 191854

15. Millstone J E, Park S, Shuford K L, Qin L, Schatz G C and Mirkin C A 2005 J. Am. Chem. Soc. 1275312

16. Ha T W, Koo H J and Chung B H 2007 J. Phys. Chem. C 1111123
17. Sun X, Dong S and Wang E 2004 Angew. Chem. Int. Ed. 436360

18. Shao Y, Jin Y and Dong S 2004 Chem. Commun. 1104

19. Zhang J, Du J, Han B, Liu Z, Jiang T and Zhang Z 2006 Angew. Chem. Int. Ed. 451116

20. Zhao N, Wei Y, Sun N, Chen Q, Bai J, Zhou L, Qin Y, Li M and Qi L 2008 Langmuir 24991

21. Burt J L, Elechiguerra J L, Reyes-Gasga J, MontejanoCarrizales J M and Jose-Yucaman M 2005 J. Cryst. Growth 285681

22. Nehl C L, Liao H and Hafner J H 2006 Nano Lett. 6683

23. Yamamoto M, Kashiwagi $Y$, Sakata T, Mori $H$ and Nakamoto M 2005 Chem. Mater. 175391

24. Murphy C J, Sau T K, Gole A M, Orendorff C J, Gao J, Gou L, Hunyadi S E and Li T 2005 J. Phys. Chem. B 10913857 and the references cited there in

25. Murphy C J, Gole A M, Stone J W, Sisco P N, Alkilany A M, Goldsmith E C and Baxter S C 2008 Acc. Chem. Res. 411721

26. Skrabalak S E, Wiley B J, Kim M, Formo E V and Xia Y 2008 Nano Lett. 82077

27. Lim B, Camargo P H C and Xia Y 2008 Langmuir 24 10437

28. Rai A, Singh A, Ahmad A and Sastry M 2006 Langmuir 22736

29. Shankar S S, Rai A, Ahmad A and Sastry M 2005 Chem. Mater. 17566

30. Ahmad A, Senapati S, Khan M I, Kumar R and Sastry M 2003 Langmuir 193550

31. Mukherjee P, Ahmad A, Mandal D, Senapati S, Sainkar S R, Khan M I, Parishcha R, Ajaykumar P V, Alam M, Kumar R and Sastry M 2001 Nano Lett. 1515

32. Umar A A and Oyama M 2009 Cryst. Growth Des. 9 1146

33. Pazos-Perez N, Barbosa S, Rodríguez-Lorenzo L, Aldeanueva-Potel P, Perez-Juste J, Pastoriza-Santos I, Alvarez-Puebla R A and Liz-Marzan L M 2010 J. Phys. Chem. Lett. 124

34. Chen H M, Liu R-S and Tsai D P 2009 Cryst. Growth Des. 92079

35. Lerma-García M, Ávila M, Simó-Alfonso E F, Ríos Á and Zougagh M 2014 J. Mater. Environ. Sci. 51919

36. AL-Thabaiti S A, Hussain J I, Hashmi A A and Khan Z 2013 Can. Chem. Trans. 1238

37. Ju J-J, Lu C-X and Jan J-S 2012 J. Nanosci. Nanotechnol. 122802

38. Lee Y and Park T G 2011 Langmuir 272965

39. Lu C-C, Zhang M, Li A-J, He X-W and Yin X-B 2011 Electroanalysis 232421

40. Link S and EI-Sayed M A 1999 J. Phys. Chem. B 10 4212

41. Wu H-Y, Liu M and Huang M H 2006 J. Phys. Chem. B 11019291

42. Teng X and Yang H 2005 Nano Lett. 5885

43. Cattalini L, Ricevuto V, Orio A and Tobe M L 1968 Inorg. Chem. 755

44. Chen S, Wang Z L, Ballato J, Foulger S H and Carroll D L 2003 J. Am. Chem. Soc. 12516186

45. Wu H-L, Chen C-H and Huang M H 2009 Chem. Matter. 21110

46. Kundu S, Peng L and Liang H 2008 Inorg. Chem. 47 6344 
47. Fukuoka T, Tatehata H and Mochizuki A $2001 \mathrm{~J}$. Appl. Polym. Sci. 801687

48. Deborde M, Rabouan S, Mazellier P, Duguet J-P and Legube B 2008 Water Res. 424299
49. Atkinson A and Roy D 1995 Biochem. Biophys. Res. Commun. 210424

50. Yoshida M, Ono H, Mori Y, Chuda Y and Mori M 2002 J. Agr. Food Chem. $\mathbf{5 0} 4377$ 